\title{
Treatment patterns of melanoma by $B R A F$ mutation status in the USA from 2011 to 2017: a retrospective cohort study
}

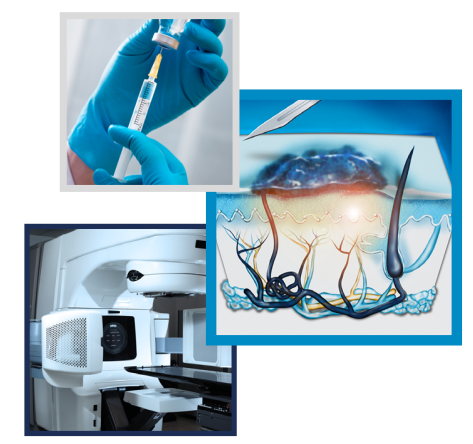

\author{
Shweta Shah*, $\neq, 1$, Leon Raskin ${ }^{\ddagger}$, David Cohan ${ }^{3}$, Omid Hamid ${ }^{4}$ \& Morganna L Freeman ${ }^{5}$ \\ ${ }^{1}$ Global Health Economics, Amgen Inc., Thousand Oaks, CA, USA \\ ${ }^{2}$ Center for Observational Research, Amgen Inc., Thousand Oaks, CA, USA \\ ${ }^{3}$ Medical Affairs, Amgen Inc., Thousand Oaks, CA, USA \\ ${ }^{4}$ The Angeles Clinic \& Research Institute, Los Angeles, CA, USA \\ ${ }^{5}$ City of Hope, Duarte, CA, USA \\ *Author for correspondence: sshah03@amgen.com \\ ¥ Authors contributed equally
}

\section{Practice points}

- Despite progress in the treatment of advanced melanoma, 5-year survival rates remain moderately low.

- Advanced melanoma with activating BRAF mutations have been associated with an increased risk of death due to rapid disease progression.

- Limited data are available regarding treatment patterns for patients with activating BRAF mutations, and changing treatment options necessitates updating published analyses of US electronic medical records.

- Our results indicate that treatment patterns for advanced melanoma dramatically changed from 2011 to 2017.

- Checkpoint inhibitors were the most commonly prescribed drug therapy overall for patients with advanced melanoma, regardless of BRAF mutation and stage, and BRAF/MEK inhibitors were the second most common.

- Other targeted therapies were rarely used, and cytokine and chemotherapy usage declined from 2011 to 2017.

- BRAF inhibitor monotherapies are being replaced by BRAF/MEK combination inhibitors.

- As new agents are approved for melanoma, ongoing surveillance of treatment patterns will be necessary to capture the changing landscape of treatment options.

Aim: To describe treatment changes from 2011 to 2017 and demographic/clinical characteristics of patients with advanced melanoma who received systemic therapy by BRAF status. Patients \& methods: Treatment patterns were evaluated in adults from the Oncology Services Comprehensive Electronic Records database who received antimelanoma systemic therapy. Results: Checkpoint inhibitors were prevailingly prescribed (66\%); usage increased from 2011 (21\%) to 2017 (84\%). BRAF/MEK inhibitors were the second most common (21\%); usage increased from $2011(6 \%)$ to $2012(18 \%)$ and stabilized until 2017 (22\%). BRAF/MEK inhibitors $(65 \%)$ and checkpoint inhibitors $(57 \%)$ were predominantly used for BRAFMut melanoma. Conclusion: Overall, checkpoint inhibitors have supplanted other therapies for advanced melanoma. Treatment shifts have occurred for $B R A F^{M u t}$ melanoma, notably increased use of checkpoint inhibitors and $\mathrm{BRAF} / \mathrm{MEK}$ combinations compared with monotherapies.

First draft submitted: 17 June 2019; Accepted for publication: 3 October 2019; Published online: 5 November 2019

Keywords: BRAF status $\bullet$ BRAF/MEK inhibitors $\bullet$ checkpoint inhibitors $\bullet$ melanoma $\bullet$ retrospective study $\bullet$ treatment patterns

Melanoma, the fifth most common cancer diagnosis in the USA accounted for $5.3 \%$ of all new cancer diagnoses and $1.5 \%$ of all cancer deaths in 2018 [1]. Advanced melanoma, a rare and serious type of skin cancer, is responsible for most skin cancer-related deaths [2], despite representing only $1 \%$ of skin cancer cases [2]. Although there has been progress in the treatment of advanced melanoma [3], 5-year survival rates remain moderately low at approximately $60 \%$ for patients presenting with stage III melanoma and $20 \%$ for those with stage IV disease [1]. In addition, cases of advanced melanoma that involve activating $B R A F$ mutations have been associated with up to a threefold

Future Medicine 
increased risk of death over a 7.6-year follow-up period due to more rapidly progressing disease, compared with melanoma without BRAF mutations [4,5].

Since 2011, the previously low survival rates of advanced melanoma have improved as a result of approvals of new immunotherapies, including ipilimumab (2011) [6], pembrolizumab (2014) [7] and nivolumab (2014) [8] that have dramatically changed the treatment landscape, response rates and other outcomes in advanced melanoma [9]. Talimogene laherparepvec, an intralesional oncolytic immunotherapy, was approved in 2015 [10] and may lead to further changes in treatment patterns in the future. In addition to the development of new BRAF/MEK inhibitors to treat $B R A F$ mutant $\left(B R A F^{M u t}\right.$ ) melanomas (vemurafenib [2011] [11], dabrafenib [2013] [12], encorafenib [2018] [13], trametinib [2013] [14], cobimetinib [2015] [15] and binimetinib [2018] [16]), there is ongoing investigation of therapeutic combinations, including triplet therapy in the management of this melanoma subtype [9].

Limited data are available regarding treatment patterns for patients with $B R A F^{M u t}$ melanoma, and the rapidly changing set of treatment options warrants updating published analyses of US electronic medical records (EMRs) [1719]. We are aware of only two other studies reporting EMR analyses in this population after 2011; however, our study had a longer follow-up time frame, had a larger patient sample size and focused on treatment distribution across multiple lines of therapy, rather than focusing on first line or survival. One prior study was an analysis of EMRs from the Flatiron database (Flatiron Health, NY, USA) that characterized treatment patterns and outcomes in patients with advanced melanoma, primarily in the first-line setting [17], and the other was a study of treatment patterns in the USA focusing on BRAF inhibitor treatment and survival outcomes specifically [18]. A third study focusing on US treatment patterns in relation to BRAF mutation testing from 2014 to 2016 was also performed; however, this analysis used patient claims data [19]. Two studies have assessed EMR data outside of the USA; one study focused on ipilimumab treatment patterns and outcomes in Europe and Australia [20], and the second study described broader treatment patterns and survival outcomes in France [21]. An observational study conducted via an online survey between August and November 2012 in Europe indicated that the most common first-line therapies for patients with BRAF $F^{\text {Mut }}$ melanoma were vemurafenib (approved in Europe in February 2012 [22]) and dacarbazine; no single treatment was preferred for the second-line setting, potentially because of lack of access to and/or reimbursement for additional treatment modalities [23].

Real-world data provide important information about how the recently approved novel agents and combination therapies are actually being used by oncologists in clinical practice with the full range of patients, including those who would have been excluded from clinical trials, and can support more informed decisions about patient care [24,25]. This study used the Oncology Services Comprehensive Electronic Records (OSCER) database (owned by Amgen Inc., CA, USA; periodic updates provided by Flatiron Health), a large repository of more than one million patients with cancer in the USA and was designed to describe the changing landscape of melanoma treatment over the 2011-2017 period. The study was intended to analyze treatment trends as new medications became available and to include the demographic and clinical characteristics of patients with advanced melanoma (predominantly stages III-IV) by BRAF mutation status. This analysis used patient-level data that contain information on biomarker status $(B R A F)$, a level of detail not often employed in previous studies for mutations known to be associated with adverse outcomes.

The primary objectives of this study were to describe demographic and clinical characteristics of patients with advanced melanoma and to describe pharmacologic treatment patterns, by line of therapy, by year and by stage, using the OSCER structured dataset. Other objectives were to describe these same characteristics according to $B R A F$ mutation status using the OSCER enhanced dataset.

\section{Patients \& methods}

Study design

We conducted a retrospective cohort study to describe treatment patterns in patients with advanced melanoma over a 6-year period beginning in 2011 (see Supplementary Data, Supplementary Figure 1). Baseline patient characteristics were collected from the 12-month period before the date of the first melanoma diagnosis (i.e., index date). Patient data were then collected from index date through death or end of study (Structured OSCER, 30 August 2017; enhanced dataset, 31 December 2016).

\section{Data source}

The OSCER collection is a proprietary database of EMRs held by cancer care providers across the USA that provides real-world clinical data representing approximately $20 \%$ of all US patients with cancer. This cloud-based 
oncology record system is used by more than 500 community oncology treatment centers and covers roughly 240 clinics, 2000 clinicians and 1,100,000 cancer patients. Two datasets were included in this analysis. The structured dataset included structured data obtained from patient-level EMRs from OSCER for patients who had received systemic therapy. The enhanced dataset included data abstracted from unstructured data obtained from physicians' notes and laboratory reports in addition to structured elements and included all patients, regardless of treatment received. The enhanced dataset also included biomarker testing results (i.e., BRAF).

\section{Patient selection}

The structured dataset included patients aged $>18$ years identified in the OSCER database who were diagnosed with advanced melanoma (International Classification of Diseases, Ninth Revision, Clinical Modification [ICD-9-CM]: 172.xx/ICD-10: C43.xx, D03.xx) between 1 January 2011 and 31 July 2017, and initiated antimelanoma therapy within 90 days of diagnosis. Patients included in the enhanced dataset are patients with advanced melanoma derived from the structured dataset and diagnosed between 1 January 2011 and 30 November 2016. For all patients, there was a 1-month minimum of follow-up to capture data. All patients had a record of at least one antimelanoma administration of chemotherapy, immunotherapy or targeted therapy. To minimize the inclusion of patients who may have received treatment outside of the EMR system, all analyses that involved lines of therapy only included patients who began treatment within 90 days of the diagnosis index date. Patients with any record of other primary malignant tumors in the year before melanoma diagnosis, with ICD codes V10.82 and Z85.820 (personal history of malignant melanoma of the skin) before the index date, aged $<19$ years (to allow $\geq 1$ year of baseline or preindex period), or with evidence of data discrepancies (e.g., diagnosis date before birth year, date of treatment initiation before diagnosis date) were excluded.

\section{Outcomes}

Treatment patterns in patients with advanced melanoma receiving systemic therapy overall, by disease stage at diagnosis, by line of therapy and by year of melanoma diagnosis (index year) were analyzed in the OSCER structured and enhanced datasets. Treatment patterns by biomarker status were also analyzed for the OSCER enhanced dataset. Demographic and clinical characteristics of patients with melanoma receiving drug treatment overall, by disease stage, by year and by line of therapy for the OSCER structured datasets are reported, as are those by biomarker status for the OSCER enhanced dataset. Stage is based on the disease reported at diagnosis; patients in a real-world treatment setting are not commonly restaged after treatment is started.

\section{Statistical analyses}

In addition to the yearly analyses, analyses over time compared the 2011-2014 and 2015-2017 periods and reported absolute differences in therapy use to capture major shifts in the treatment landscape of advanced melanoma. In the 2011-2014 period, the first immunotherapy and targeted therapy agents were approved, and in 2015-2017, newer agents, such as combinations of cobimetinib and vemurafenib were approved for melanoma treatment [3].

The frequency of melanoma treatment patterns (i.e., the order and treatment received) was counted overall, by disease stage, by line of therapy and by year. First, second and third lines of melanoma therapy were identified by disease stage, $B R A F$ mutation status and by year. Treatments were described by disease stage, by line of therapy, by year of melanoma diagnosis and by biomarker (in enhanced dataset). The time trends of therapy were described by year of melanoma diagnosis during the study period (i.e., 2011-2017). Demographic and clinical characteristics were summarized by disease stage, line of therapy, year of index diagnosis and biomarker status (continuous variables were summarized as mean and standard deviation; categorical variables were summarized as counts and percentages). Flatiron Health did not participate in the analyses of these data.

\section{Results}

Patients

Overall, 2628 patients listed in the OSCER database with melanoma who received drug therapy from 2011 to 2017 and who initiated therapy for melanoma within 90 days of diagnosis were included in the structured dataset. The enhanced dataset comprised 1687 patients with known BRAF mutation status from 2011 to 2016, 1161 of whom were patients with stage III (41\% [688/1687]) or IV (28\% [473/1687]) melanoma (Table 1, enhanced dataset). Demographic and clinical characteristics are shown in Table 1 . In general, most patients were aged $\geq 40$ years (structured dataset, 94\% [2460/2628]; enhanced dataset, 93\% [1564/1687]) and had stage III or IV disease at 
Table 1. Patient demographics and baseline clinical characteristics.

\begin{tabular}{|c|c|c|c|c|c|}
\hline \multirow[t]{2}{*}{ Characteristic } & \multicolumn{2}{|c|}{ Structured Dataset (2011-2017) ${ }^{\dagger}$} & \multicolumn{3}{|c|}{ Enhanced dataset (2011-2016) ${ }^{\dagger}$} \\
\hline & $\begin{array}{l}\text { OSCER 2011-2014 } \\
(\mathrm{n}=1051)\end{array}$ & $\begin{array}{l}\text { OSCER 2015-2017 } \\
(n=1577)\end{array}$ & Overall $(n=1687)$ & $\operatorname{BRAF}^{\text {Mut }}(\mathrm{n}=557)$ & $B R A F^{W t}(n=1130)$ \\
\hline Male & $657(62.5)$ & $1040(66.0)$ & $1136(67.3)$ & $367(65.9)$ & $769(68.1)$ \\
\hline $18-39$ & $85(8.1)$ & $83(5.3)$ & $123(7.3)$ & $50(9.0)$ & $73(6.5)$ \\
\hline $40-64$ & $483(46.0)$ & $597(37.9)$ & $727(43.1)$ & $288(51.7)$ & $439(38.9)$ \\
\hline \multicolumn{6}{|l|}{ Line of therapy } \\
\hline 1 & $1051(100.0)$ & $1577(100.0)$ & $1324(78.5)$ & $495(88.9)$ & $829(73.4)$ \\
\hline 2 & $369(35.1)$ & $512(32.5)$ & $435(25.8)$ & $203(36.5)$ & $232(20.5)$ \\
\hline 3 & $158(15.0)$ & $166(10.5)$ & $128(7.6)$ & $73(13.1)$ & $55(4.9)$ \\
\hline $4+$ & $84(8.0)$ & $49(3.1)$ & $51(3.0)$ & $26(4.7)$ & $25(2.2)$ \\
\hline 2 & $38(3.6)$ & $126(8.0)$ & $85(5.0)$ & $25(4.5)$ & $60(5.3)$ \\
\hline 3 & $16(1.5)$ & $39(2.5)$ & $24(1.4)$ & $10(1.8)$ & $14(1.2)$ \\
\hline 4 & $1(0.1)$ & $4(0.3)$ & $7(0.4)$ & $3(0.5)$ & $4(0.4)$ \\
\hline Missing & $598(56.9)$ & $529(33.5)$ & $721(42.7)$ & $243(43.6)$ & $478(42.3)$ \\
\hline \multicolumn{6}{|l|}{ Disease stage } \\
\hline I & $4(0.4)$ & $6(0.4)$ & $90(5.3)$ & $37(6.6)$ & $53(4.7)$ \\
\hline II & $30(2.9)$ & $34(2.2)$ & $251(14.9)$ & $76(13.6)$ & $175(15.5)$ \\
\hline III & $93(8.9)$ & $147(9.3)$ & $688(40.8)$ & $191(34.3)$ & $497(44.0)$ \\
\hline IV & $256(24.4)$ & $544(34.5)$ & $473(28.0)$ & $195(35.0)$ & $278(24.6)$ \\
\hline Missing & $668(63.6)$ & $846(53.7)$ & $185(11.0)$ & $58(10.4)$ & $127(11.2)$ \\
\hline Other & $25(2.4)$ & $123(7.8)$ & $325(19.3)$ & $134(24.1)$ & $191(16.9)$ \\
\hline \multicolumn{6}{|c|}{ Number of sites of metastases } \\
\hline 1 & $18(1.7)$ & $235(14.9)$ & $422(25.0)$ & $158(28.4)$ & $264(23.4)$ \\
\hline 2 & $6(0.6)$ & $76(4.8)$ & $179(10.6)$ & $81(14.5)$ & $98(8.7)$ \\
\hline $3+$ & $20(1.9)$ & $99(6.3)$ & $71(4.2)$ & $29(5.2)$ & $42(3.7)$ \\
\hline NA & $1007(95.8)$ & $1167(74.0)$ & $1015(60.2)$ & $289(51.9)$ & $726(64.3)$ \\
\hline
\end{tabular}

the time of first diagnosis (structured dataset, 40\% [1040/2628], missing, 58\% [1514/2628]; enhanced dataset, 69\% [1161/1687], missing, 11\% [185/1687]); slightly more than half (structured dataset, 51\% [1338/2628]; enhanced dataset, 36\% [614/1687]) had received at least two lines of systemic therapy. A greater incidence of lymph node metastasis was observed in patients with $B R A F^{M u t}$ tumors (13\% [72/557]) compared with patients with $B R A F^{W T}$ tumors (11\% [124/1130]). 
Table 2. Melanoma treatment by year for all patients (structured dataset).

\begin{tabular}{|c|c|c|c|c|c|c|c|c|}
\hline Drug agent & $\begin{array}{l}\text { Overall } \\
(\mathrm{N}=2628)\end{array}$ & $\begin{array}{l}2011 \\
(n=182)\end{array}$ & $\begin{array}{l}2012 \\
(n=348)\end{array}$ & $\begin{array}{l}2013 \\
(n=365)\end{array}$ & $\begin{array}{l}2014 \\
(n=473)\end{array}$ & $\begin{array}{l}2015 \\
(n=609)\end{array}$ & $\begin{array}{l}2016 \\
(n=1083)\end{array}$ & $\begin{array}{l}2017 \\
(n=1008)\end{array}$ \\
\hline Checkpoint inhibitors $^{\dagger}$ & $1722(65.5)$ & $38(20.9)$ & $101(29.0)$ & $130(35.6)$ & $216(45.7)$ & $365(59.9)$ & $840(77.6)$ & $845(83.8)$ \\
\hline - Ipilimumab & $729(27.7)$ & 38 (20.9) & $101(29.0)$ & $130(35.6)$ & $204(43.1)$ & $154(25.3)$ & $176(16.3)$ & $138(13.7)$ \\
\hline - Pembrolizumab & $496(18.9)$ & - & - & - & $34(7.2)$ & $130(21.3)$ & $262(24.2)$ & 308 (30.6) \\
\hline - Nivolumab & $410(15.6)$ & - & - & - & - & $91(14.9)$ & $248(22.9)$ & $247(24$. \\
\hline - Ipilimumab + nivolumab & $388(14.8)$ & - & - & - & - & $48(7.9)$ & $240(22.2)$ & $234(23.2)$ \\
\hline BRAF/MEK inhibitors ${ }^{\ddagger}$ & $561(21.3)$ & $10(5.5)$ & $62(17.8)$ & $83(22.7)$ & $107(22.6)$ & $115(18.9)$ & $232(21.4)$ & $219(21.7)$ \\
\hline - Dabrafenib & $51(1.9)$ & - & - & $7(1.9)$ & $13(2.7)$ & $4(0.7)$ & $21(1.9)$ & $18(1.8)$ \\
\hline - Trametinib & $37(1.4)$ & - & - & $3(0.8)$ & $10(2.1)$ & $6(1.0)$ & $12(1.1)$ & $13(1.3)$ \\
\hline - Vemurafenib & $192(7.3)$ & $10(5.5)$ & $62(17.8)$ & $75(20.5)$ & $47(9.9)$ & $32(5.3)$ & $33(3.0)$ & $22(2.2)$ \\
\hline - Cobimetinib & $6(0.2)$ & - & - & - & - & - & $3(0.3)$ & $4(0.4)$ \\
\hline - Dabrafenib + trametinib & $324(12.3)$ & - & - & $4(1.1)$ & $55(11.6)$ & $79(13.0)$ & $161(14.9)$ & $153(15.2)$ \\
\hline - Cobimetinib + vemurafenib & $67(2.5)$ & - & - & - & - & $4(0.7)$ & $39(3.6)$ & $45(4.5)$ \\
\hline Other targeted therapy ${ }^{\S}$ & $30(1.1)$ & - & $6(1.7)$ & $5(1.4)$ & $10(2.1)$ & $11(1.8)$ & $15(1.4)$ & $14(1.4)$ \\
\hline Cytokines & $504(19.2)$ & $82(45.1)$ & $147(42.2)$ & $130(35.6)$ & $143(30.2)$ & $130(21.3)$ & $79(7.3)$ & $38(3.8)$ \\
\hline - Interferon $\alpha-2 \beta$ & $502(19.1)$ & $82(45.1)$ & $147(42.2)$ & $129(35.3)$ & $142(30.0)$ & $130(21.3)$ & $78(7.2)$ & $38(3.8)$ \\
\hline - Peginterferon $\alpha-2 \beta$ & $142(5.4)$ & $19(10.4)$ & $40(11.5)$ & $44(12.1)$ & $45(9.5)$ & $42(6.9)$ & $26(2.4)$ & $10(1.0)$ \\
\hline - Aldesleukin & $4(0.2)$ & - & - & $1(0.3)$ & $1(0.2)$ & $1(0.2)$ & $1(0.1)$ & - \\
\hline Chemotherapy & $339(12.9)$ & $65(35.7)$ & $73(21.0)$ & $71(19.5)$ & $68(14.4)$ & $57(9.4)$ & $46(4.2)$ & $42(4.2)$ \\
\hline - Temozolomide & $147(5.6)$ & $40(22.0)$ & $41(11.8)$ & $35(9.6)$ & $26(5.5)$ & $12(2.0)$ & $9(0.8)$ & $14(1.4)$ \\
\hline - Fluorouracil & $73(2.8)$ & $4(2.2)$ & $7(2.0)$ & $9(2.5)$ & $20(4.2)$ & $23(3.8)$ & $13(1.2)$ & $10(1.0)$ \\
\hline - Carboplatin & $73(2.8)$ & $9(4.9)$ & $15(4.3)$ & $16(4.4)$ & $12(2.5)$ & $10(1.6)$ & $17(1.6)$ & $14(1.4)$ \\
\hline - PaclitaxelI & $95(3.6)$ & $11(6.0)$ & $18(5.2)$ & $21(5.8)$ & $20(4.2)$ & $16(2.6)$ & $19(1.8)$ & $18(1.8)$ \\
\hline - Dacarbazine & $49(1.9)$ & $12(6.6)$ & $10(2.9)$ & $10(2.7)$ & $7(1.5)$ & $6(1.0)$ & $3(0.3)$ & $6(0.6)$ \\
\hline - Cisplatin & $25(1.0)$ & $2(1.1)$ & $11(3.2)$ & $2(0.5)$ & $2(0.4)$ & $4(0.7)$ & $3(0.3)$ & $5(0.5)$ \\
\hline - Cyclophosphamide & $2(0.1)$ & - & - & $1(0.3)$ & $2(0.4)$ & $1(0.2)$ & $1(0.1)$ & $1(0.1)$ \\
\hline - Other chemotherapy & $1(0.04)$ & - & - & - & - & - & $1(0.1)$ & - \\
\hline \multicolumn{9}{|c|}{$\begin{array}{l}\text { Data are presented as } n(\%) \text {. } \\
\text { †FDA approval dates of checkpoint inhibitors for melanoma treatment: ipilimumab, March 2011; pembrolizumab, September 2014; nivolumab, December } 2014 . \\
\text { †FDA approval dates of BRAF/MEK inhibitors for melanoma treatment: dabrafenib, May 2013; trametinib, May 2013; vemurafenib, August } 2011 \text {; cobimetinib, November } 2015 . \\
\text { \$Other targeted therapies included bevacizumab, bortezomib, rituximab, and ruxolitinib phosphate. } \\
\mathbb{I}_{\text {And Paclitaxel protein bound. }} \\
\text { FDA: US Food and Drug Administration. }\end{array}$} \\
\hline
\end{tabular}

\section{Treatment patterns}

Therapies for advanced melanoma included checkpoint inhibitors (66\% [1722/2628]), BRAF/MEK inhibitors (21\% [561/2628]), cytokines (19\% [504/2628]), and chemotherapy (13\% [339/2628]).

\section{Treatment patterns over the period evaluated (structured dataset)}

Overall, the use of checkpoint inhibitor treatments increased from 21\% (38/182) in 2011 to 84\% (845/1008) in 2017 (Figure 1A). Ipilimumab remained the sole US FDA-approved checkpoint inhibitor until 2014, at which point its use as a monotherapy decreased with the increased use of other monotherapy inhibitors and combination therapy of ipilimumab plus nivolumab (Table 2). The overall use of BRAF and MEK inhibitors remained fairly stable from 2013 to 2017 (approximately 20\%); however, by 2017, the use of vemurafenib monotherapy (2\% [22/1008]) was largely replaced by the BRAF/MEK inhibitor combinations, mainly dabrafenib + trametinib and less frequently, cobimetinib + vemurafenib (15\% [153/1008] and 5\% [45/1008]; Table 2). Additionally, cytokine and chemotherapy use declined over the period evaluated (45\% [82/182] to 4\% [38/1008] and 36\% [65/182] to $4 \%$ [ $42 / 1008]$, respectively); however, the use of high-dose interleukin-2 (IL-2) (i.e., aldesleukin) remained low and constant over the course of the study $(<1 \%)$.

For patients with stage III disease $(\mathrm{n}=240)$, use of checkpoint inhibitors increased from $13 \%(3 / 24)$ to $87 \%$ (93/107) from 2011 to 2017, corresponding with a decreased use of cytokines from 96\% (23/24) in 2011 to 11\% (12/107) in 2017 (see Supplementary Data, Supplementary Table 1). Use of BRAF/MEK inhibitors was 
(A)

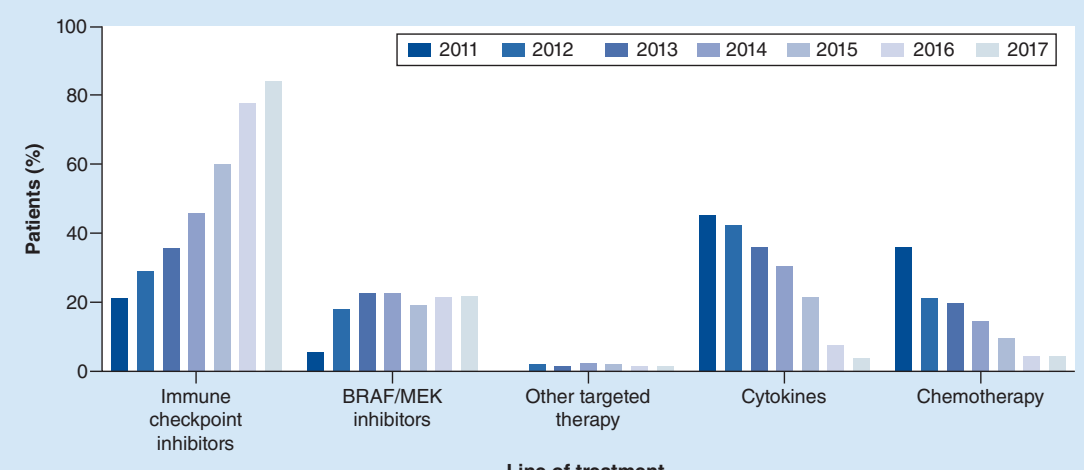

(B)

Line of treatment

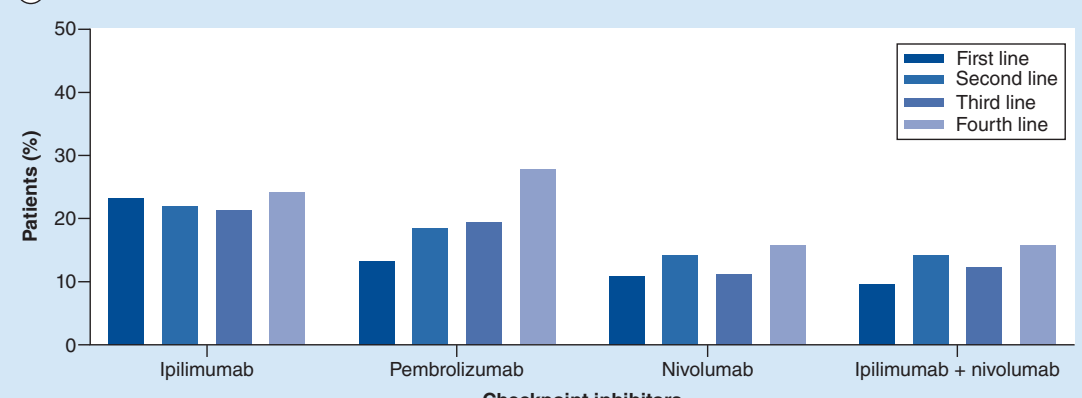

(c)

Checkpoint inhibitors

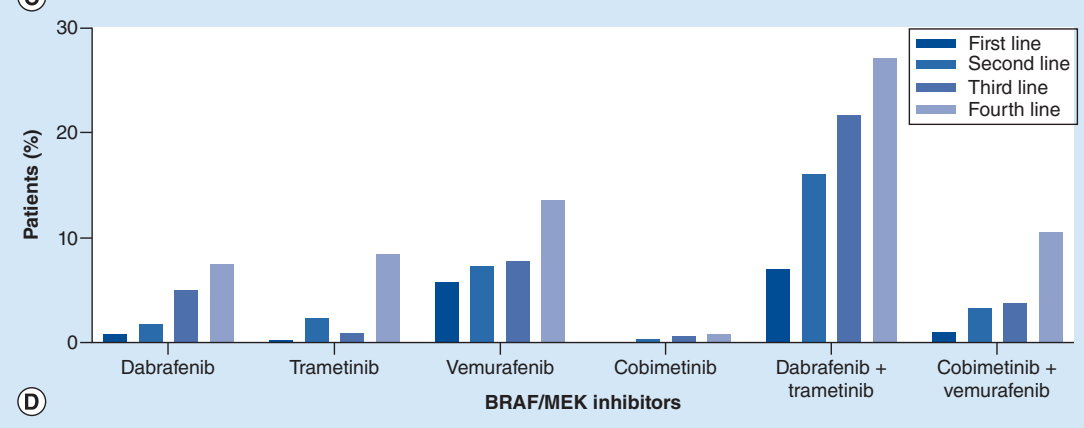

(D)

BRAF/MEK inhibitors

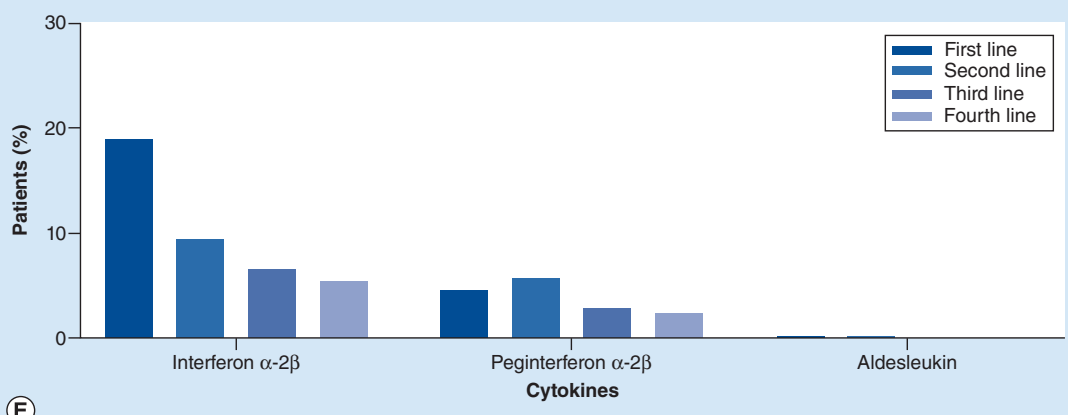

(E)

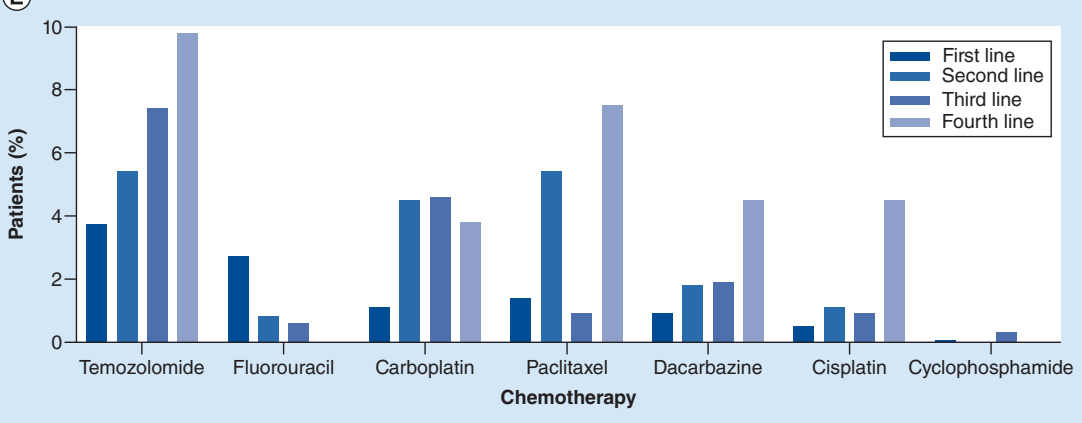

Figure 1. Treatment patterns. By (A) year and line of treatment for (B) checkpoint inhibitors, (C) BRAF/MEK inhibitors, (D) cytokines and (E) chemotherapy in the Structured Dataset. 
initially limited (3\% [1/34] in 2012) but increased over the period evaluated (18\% [19/107] in 2017). Use of chemotherapy was minimal (3\% [7/240] overall).

For patients with stage IV disease $(\mathrm{n}=800)$, use of checkpoint inhibitors also increased from 2011 to 2017, from 35\% (15/43) to $90 \%$ (333/372; see Supplementary Data, Supplementary Table 2). Additionally, the use of BRAF/MEK inhibitors increased from $9 \%(4 / 43)$ in 2011 to $24 \%(88 / 372)$ in 2017. Cytokine use decreased from $9 \%(4 / 43)$ in 2011 to $1 \%(1 / 372)$ in 2015 and $0 \%$ in 2016 and 2017. Chemotherapy use also decreased from 53\% (23/43) in 2011 to 3\% (13/372) in 2017.

\section{Treatment patterns by line of therapy (structured dataset)}

Checkpoint inhibitors were the most commonly administered treatment for the first line of therapy (57\% [1498/2628]; see Supplementary Data, Supplementary Table 3) and across later lines of therapy (ranging from $59 \%$ [190/324] to 66\% [88/133]). Of note, the use of pembrolizumab (13\% [349/2628] in first line to $28 \%$ [37/133] in fourth line) and ipilimumab/nivolumab (10\% [255/2628] in first line to $16 \%$ [21/133] in fourth line) increased in later lines of therapy (Figure 1B). The next most commonly used treatments overall were BRAF/MEK inhibitors (21\% [561/2628]; Figure 1C) and cytokines (19\% [504/2628]; Figure 1D), respectively, followed by chemotherapy (13\% [339/2628]; Figure 1E). When BRAF/MEK inhibitors were used, vemurafenib use was higher in first-line therapy (39\% [149/381] of BRAF/MEK inhibitor treatments) compared with fourth-line therapy (28\% [18/64]), whereas the use of other BRAF and MEK inhibitors increased in later lines of therapy. Cytokine use was predominantly observed as first (19\% [499/2628]) and second lines (10\% [84/881]) of therapy compared with later lines (5-6\%) of therapy.

For patients with stage III disease $(\mathrm{n}=367)$, checkpoint inhibitor use increased with later lines of therapy (first line, 53\% [128/240]; fourth line, 86\% [12/14]; Table 3). Use of BRAF/MEK inhibitors also increased with later lines of therapy (first line, 4\% [10/240]; fourth line, 43\% [6/14]). Conversely, cytokine use decreased from $42 \%$ $(101 / 240)$ in the first-line setting to $0 \%$ in the fourth-line setting, suggesting an absolute decrease in their use over time.

In contrast to patients with stage III disease, use of checkpoint inhibitors decreased across later lines of therapy for patients with stage IV disease $(\mathrm{n}=1172)$, and cytokine therapy use remained low throughout all lines of therapy (Table 3). BRAF/MEK inhibitor use and chemotherapy were more prevalent in patients with stage IV disease; for both, use increased across later lines of therapy.

\section{Treatment patterns by biomarker status (enhanced dataset)}

For patients with $B R A F^{M u t}$ tumors, checkpoint inhibitor use was higher overall (see Supplementary Data, Supplementary Figure 2) and higher in patients with stage III disease $(62 \%$ [119/191]) than in those with stage IV disease (52\% [102/195]). In contrast, for patients with $B R A F^{W t}$ tumors, checkpoint inhibitor use overall was lower in patients with stage III disease (52\% [258/497]) than in those with stage IV disease (90\% [250/278]; Figure 2A; Table 4). BRAF/MEK inhibitors were the most commonly used agents for both stage III and IV BRAF ${ }^{M u t}$ disease overall (65\% [249/386]). Furthermore, BRAF/MEK inhibitors were used in a higher percentage of patients with $B R A F^{M u t}$ stage IV disease (79\% [154/195]) than those with BRAF $F^{M u t}$ stage III disease (50\% [95/191]; Figure 2B). Cytokines were more commonly used for patients with both $B R A F^{M u t}$ and $B R A F^{W t}$ stage III disease (37\% [70/191]; $46 \%$ [228/497]) versus stage IV disease (1\% [2/195]; 4\% [10/278]; Figure 2C). Chemotherapy use was similar for patients with $B R A F^{M u t}$ stage III $(5 \%$ [10/191]) or stage IV $(6 \%$ [11/195]) disease; use was slightly higher in patients with $B R A F^{W t}$ stage IV disease (18\% [51/278]) versus stage III disease (8\% [39/497]; Figure 2D).

Stratified data from 2011 to 2013 for patients with either $B R A F^{M u t}$ or $B R A F^{W t}$ stage III or IV disease are limited (see Supplementary Data, Supplementary Tables 4-7). From 2014 to 2016, BRAF/MEK inhibitors and checkpoint inhibitors were the most commonly used therapies across all lines of therapy for patients with $B R A F^{M u t}$ stage III or IV disease (see Supplementary Data, Supplementary Tables $8 \&$ 9). For patients with $B R A F^{W t}$ stage III or IV disease, checkpoint inhibitors and chemotherapy were the most commonly used treatments from 2014 to 2016 across all lines of therapy (see Supplementary Data, Supplementary Tables $10 \& 11$ ). Although uncommon, use of $\mathrm{BRAF} / \mathrm{MEK}$ inhibitors was observed in patients with $B R A F^{W t}$ tumors, mostly in patients with stage III disease (see Supplementary Data, Supplementary Tables $10 \& 11$ ). Use of cytokines and targeted therapies other than BRAF and MEK inhibitors was sporadic, regardless of $B R A F$ mutation status, disease stage or time period. 
(A)

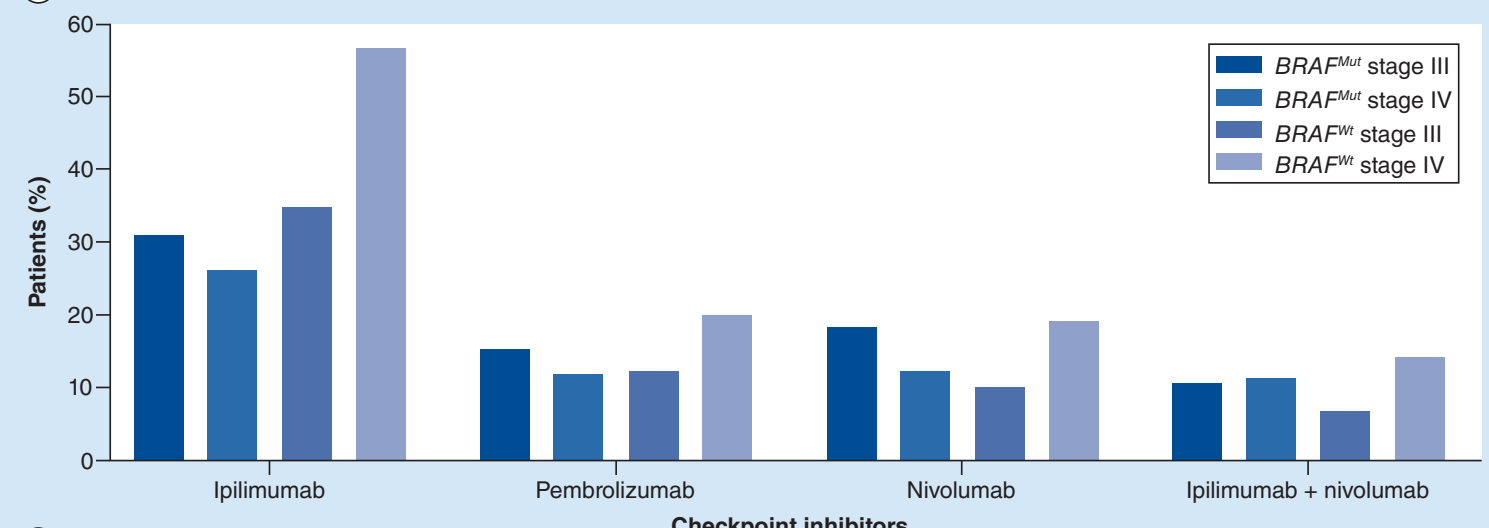

(B)

Checkpoint inhibitors

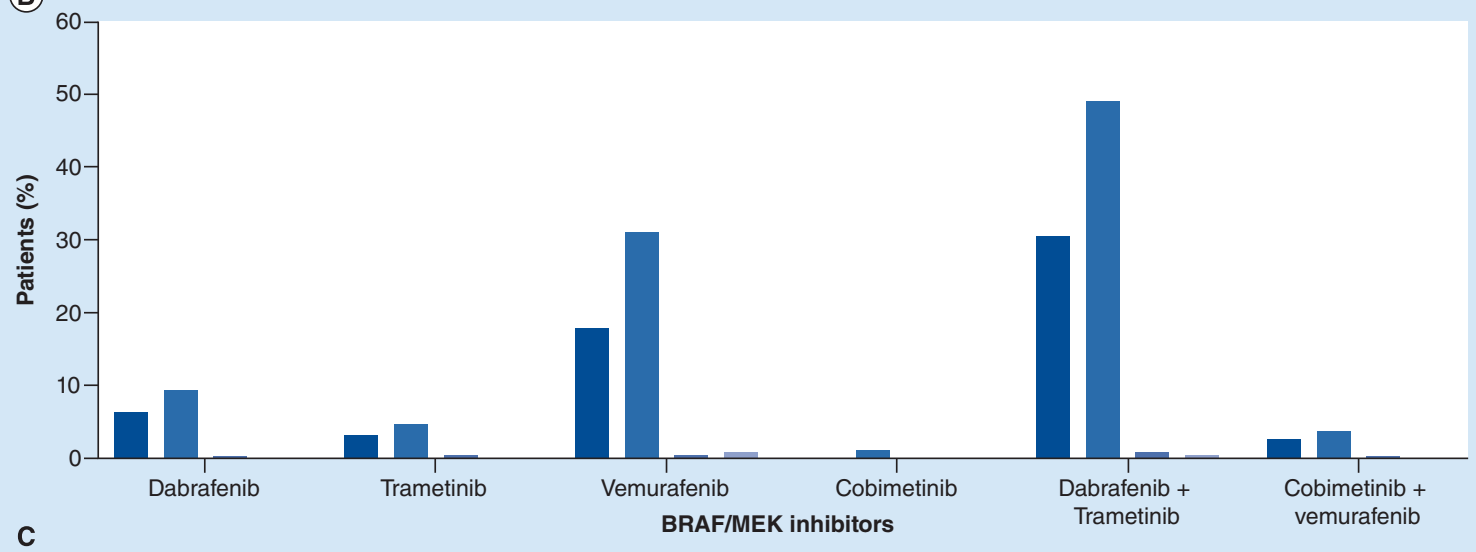

C
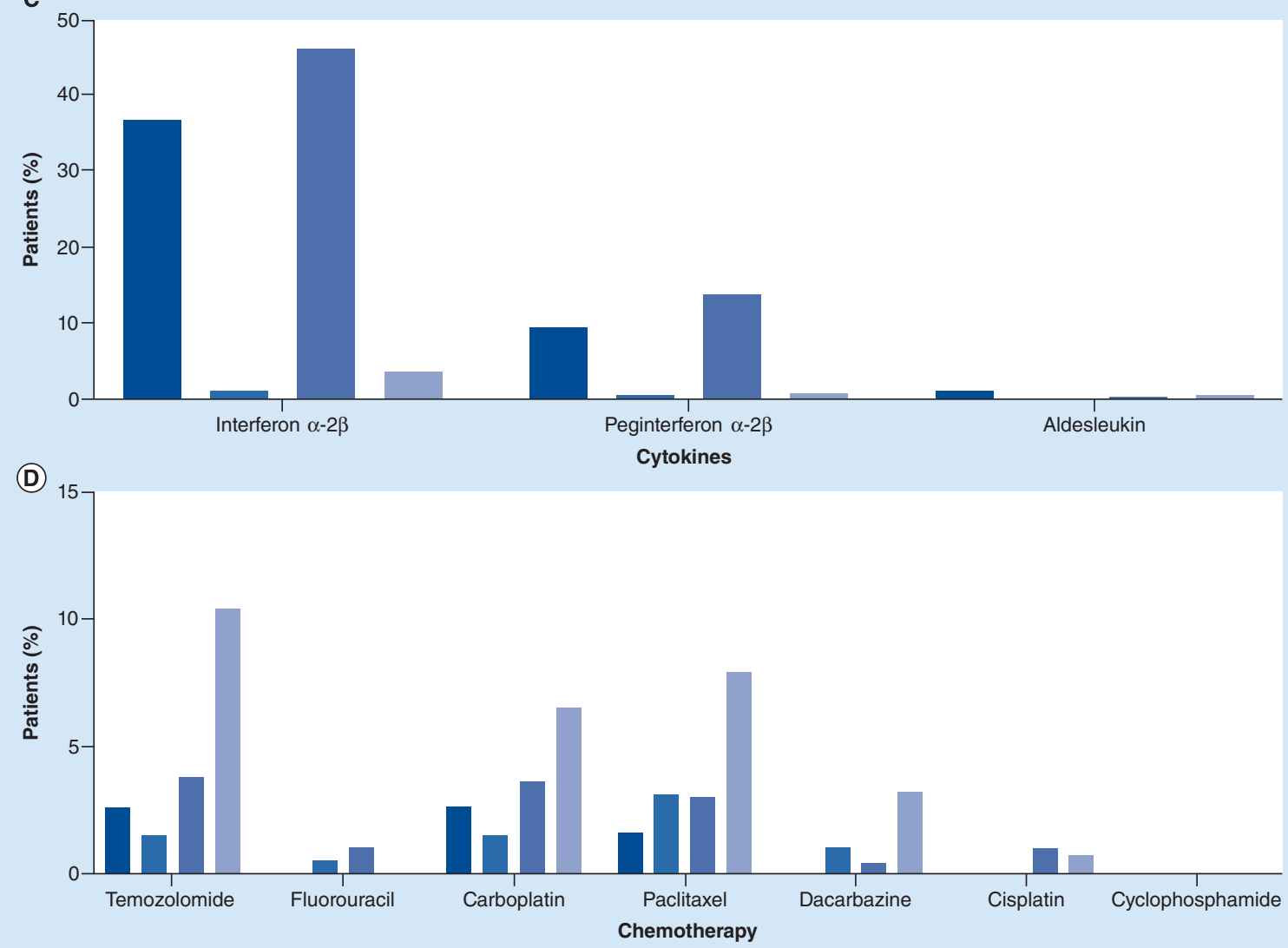

Figure 2. Treatment patterns. For (A) checkpoint inhibitors, (B) BRAF/MEK inhibitors, (C) cytokines and (D) chemotherapy by disease stage and $B R A F$ status in the enhanced dataset. 
Table 3. Melanoma treatment by line of therapy for patients by disease stage (structured dataset).

\begin{tabular}{|c|c|c|c|c|c|c|c|c|}
\hline \multirow[t]{2}{*}{ Drug agent } & \multicolumn{4}{|c|}{ Stage III $(n=367)$} & \multicolumn{4}{|c|}{ Stage IV $(n=1172)$} \\
\hline & $1 \mathrm{~L}(\mathrm{n}=240)$ & $2 L(n=83)$ & $3 L(n=30)$ & $4 L(n=14)$ & $1 \mathrm{~L}(\mathrm{n}=800)$ & $2 L(n=261)$ & $3 L(n=85)$ & $4 \mathrm{~L}(n=26)$ \\
\hline Checkpoint inhibitors $^{\dagger}$ & $128(53.3)$ & $58(69.9)$ & $21(70.0)$ & $12(85.7)$ & $590(73.8)$ & $183(70.1)$ & $45(52.9)$ & $16(61.5)$ \\
\hline - Ipilimumab & $105(43.8)$ & $34(41.0)$ & $13(43.3)$ & $8(57.1)$ & $176(22.0)$ & $53(20.31)$ & $18(21.2)$ & $3(11.5)$ \\
\hline - Pembrolizumab & $11(4.6)$ & $13(15.7)$ & $4(13.3)$ & $2(14.3)$ & $143(17.9)$ & $48(18.4)$ & $13(15.3)$ & $9(34.6)$ \\
\hline - Nivolumab & $6(2.5)$ & $7(8.4)$ & $1(3.3)$ & $2(14.3)$ & $133(16.6)$ & $45(17.2)$ & $8(9.4)$ & $5(19.2)$ \\
\hline - Ipilimumab + nivolumab & $6(2.5)$ & $4(4.8)$ & $3(10.0)$ & $2(14.3)$ & $138(17.3)$ & $51(19.5)$ & $10(11.8)$ & $2(7.7)$ \\
\hline BRAF/MEK inhibitors ${ }^{\ddagger}$ & $10(4.2)$ & $12(14.5)$ & $10(33.3)$ & $6(42.9)$ & $143(17.9)$ & $104(39.9)$ & 41 (48.2) & $12(46.2)$ \\
\hline - Dabrafenib & - & $1(1.2)$ & $1(3.3)$ & - & $8(1.0)$ & $5(1.9)$ & $5(5.9)$ & $4(15.4)$ \\
\hline - Trametinib & - & - & - & $2(14.3)$ & $2(0.3)$ & $10(3.8)$ & $1(1.2)$ & $2(7.7)$ \\
\hline - Vemurafenib & $4(1.7)$ & $3(3.6)$ & $1(3.3)$ & - & $51(6.4)$ & $22(8.4)$ & $5(5.9)$ & $1(3.9)$ \\
\hline - Cobimetinib & - & - & - & - & - & $2(0.8)$ & $1(1.2)$ & - \\
\hline - Dabrafenib + trametinib & $3(1.3)$ & $5(6.0)$ & $8(26.7)$ & $5(35.7)$ & $71(8.9)$ & $56(21.5)$ & $27(31.8)$ & $6(23.1)$ \\
\hline - Cobimetinib + vemurafenib & $3(1.3)$ & $3(3.6)$ & - & $1(7.1)$ & $13(1.6)$ & $14(5.4)$ & $5(5.9)$ & $4(15.4)$ \\
\hline Other targeted therapy $\S$ & - & - & - & - & $2(0.3)$ & $2(0.8)$ & - & - \\
\hline Cytokines & $101(42.1)$ & $17(20.5)$ & $2(6.7)$ & - & $10(1.3)$ & $3(1.2)$ & $1(1.2)$ & - \\
\hline - Interferon $\alpha-2 \beta$ & $101(42.1)$ & $17(20.5)$ & $2(6.7)$ & - & $10(1.3)$ & $3(1.2)$ & $1(1.2)$ & - \\
\hline - Peginterferon $\alpha-2 \beta$ & $26(10.8)$ & $11(13.3)$ & $1(3.3)$ & - & $2(0.3)$ & $1(0.4)$ & - & - \\
\hline - Aldesleukin & - & - & - & - & - & - & - & - \\
\hline Chemotherapy & $1(0.4)$ & $4(4.8)$ & $1(3.3)$ & $2(14.3)$ & $59(7.4)$ & $41(15.7)$ & $20(23.5)$ & $6(23.1)$ \\
\hline - Temozolomide & - & $2(2.4)$ & $1(3.3)$ & $1(7.1)$ & $33(4.1)$ & $15(5.8)$ & $10(11.8)$ & $5(19.2)$ \\
\hline - Fluorouracil & $1(0.4)$ & - & - & - & - & - & - & - \\
\hline - Carboplatin & - & - & - & - & $10(1.3)$ & $19(7.3)$ & $4(4.7)$ & - \\
\hline - Paclitaxel I & - & - & - & - & $12(1.5)$ & $19(7.3)$ & $8(9.4)$ & $2(7.7)$ \\
\hline - Dacarbazine & - & $2(2.4)$ & - & $2(14.3)$ & $10(1.3)$ & $6(2.3)$ & $4(4.7)$ & $1(3.9)$ \\
\hline - Cisplatin & - & - & - & $1(7.1)$ & $5(0.6)$ & $4(1.5)$ & $1(1.2)$ & $1(3.9)$ \\
\hline - Cyclophosphamide & - & - & - & - & - & - & - & - \\
\hline - Other chemotherapy & - & - & - & - & - & - & - & - \\
\hline \multicolumn{9}{|c|}{$\begin{array}{l}\text { Therapies were not exclusive; patients may have received multiple therapies. } \\
\text { †FDA approval dates of checkpoint inhibitors for melanoma treatment: ipilimumab, March 2011; pembrolizumab, September 2014; nivolumab, December } 2014 . \\
\text { ¥FDA approval dates of BRAF/MEK inhibitors for melanoma treatment: dabrafenib, May 2013; trametinib, May 2013; vemurafenib, August } 2011 \text {; cobimetinib, November } 2015 . \\
\text { §Other targeted therapies included bevacizumab, bortezomib, rituximab, and ruxolitinib phosphate. } \\
\mathbb{I}_{\text {And Paclitaxel protein bound. }}\end{array}$} \\
\hline
\end{tabular}

\section{Discussion}

Data from real-world analyses such as the ones reported here could further augment clinician knowledge and help guide treatment selections. In this analysis, checkpoint inhibitors were the most commonly prescribed drug therapy overall for patients with advanced melanoma, regardless of $B R A F$ mutation and stage. Additionally, checkpoint inhibitors, BRAF/MEK inhibitors and chemotherapy were the predominant choices for later-line therapies after first-line treatment for stage IV disease. Other targeted therapies and cytokines, including high-dose IL-2, were less prescribed.

Most melanoma patients with $B R A F^{M u t}$ tumors (65\%) were initially prescribed BRAF/MEK inhibitors. Notably, the use of later FDA-approved BRAF (i.e., dabrafenib) and MEK (trametinib and cobimetinib) inhibition for the treatment of advanced melanoma increased compared with the use of the first BRAF inhibitor monotherapy (vemurafenib) approved in 2011. In patients with BRAF mutations, BRAF/MEK inhibitors were more commonly prescribed for treatment in stage IV than in stage III melanoma. This could reflect changes in the treatment landscape because BRAF/MEK inhibitors may not have been approved for use when the patients included in this report were diagnosed and received first-line treatment. The use of other targeted therapies (i.e., rituximab, bevacizumab, bortezomib and ruxolitinib phosphate) was sparse and occurred mostly as single-agent use in the first- and second-line settings for patients with stage IV melanoma or those who did not meet the criteria for receiving one of the other therapeutic options. Although it has also been suggested that targeted therapy with 


\begin{tabular}{|c|c|c|c|c|c|c|c|}
\hline \multirow[t]{2}{*}{ Drug agent } & \multirow{2}{*}{$\begin{array}{l}\text { Overall } \\
(\mathrm{N}=1161)\end{array}$} & \multicolumn{3}{|c|}{ BRAF $F^{\text {Mut }}$} & \multicolumn{3}{|c|}{$B R A F^{W t}$} \\
\hline & & Total $(n=386)$ & $\begin{array}{l}\text { Stage III } \\
(n=191)\end{array}$ & $\begin{array}{l}\text { Stage IV } \\
(n=195)\end{array}$ & Total $(n=775)$ & $\begin{array}{l}\text { Stage III } \\
(n=497)\end{array}$ & $\begin{array}{l}\text { Stage IV } \\
(n=278)\end{array}$ \\
\hline Checkpoint inhibitors $^{\dagger}$ & $729(62.8)$ & $221(57.3)$ & $119(62.3)$ & $102(52.3)$ & $508(65.5)$ & $258(51.9)$ & $250(89.9)$ \\
\hline - Ipilimumab & $441(38)$ & $110(28.5)$ & $59(30.9)$ & $51(26.2)$ & $331(42.7)$ & $173(34.8)$ & $158(56.8)$ \\
\hline - Pembrolizumab & $168(14.5)$ & $52(13.5)$ & $29(15.2)$ & $23(11.8)$ & $116(15)$ & $61(12.3)$ & $55(19.8)$ \\
\hline - Nivolumab & $162(14.0)$ & $59(15.3)$ & $35(18.3)$ & $24(12.3)$ & $103(13.3)$ & $50(10.1)$ & $53(19.1)$ \\
\hline - Ipilimumab + nivolumab & $115(9.9)$ & $42(10.9)$ & $20(10.5)$ & $22(11.3)$ & $73(9.4)$ & $34(6.8)$ & $39(14.0)$ \\
\hline BRAF/MEK inhibitors ${ }^{\ddagger}$ & $262(22.6)$ & $249(64.5)$ & $95(49.7)$ & $154(79.0)$ & $13(1.7)$ & $10(2.0)$ & $3(1.1)$ \\
\hline - Dabrafenib & $31(2.7)$ & $30(7.8)$ & $12(6.3)$ & $18(9.2)$ & $1(0.1)$ & $1(0.2)$ & - \\
\hline - Trametinib & $17(1.5)$ & $15(3.9)$ & $6(3.1)$ & $9(4.6)$ & $2(0.3)$ & $2(0.4)$ & - \\
\hline - Vemurafenib & $98(8.4)$ & $94(24.4)$ & $34(17.8)$ & $60(30.8)$ & $4(0.5)$ & $2(0.4)$ & $2(0.7)$ \\
\hline - Cobimetinib & $2(0.2)$ & $2(0.5)$ & - & $2(1.0)$ & - & - & - \\
\hline - Dabrafenib + trametinib & $158(13.6)$ & $153(39.6)$ & $58(30.4)$ & $95(48.7)$ & $5(0.6)$ & $4(0.8)$ & $1(0.4)$ \\
\hline - Cobimetinib + vemurafenib & $13(1.1)$ & $12(3.1)$ & $5(2.6)$ & $7(3.6)$ & $1(0.1)$ & $1(0.2)$ & - \\
\hline Other targeted therapy ${ }^{\S}$ & $15(1.3)$ & $8(2.1)$ & $4(2.1)$ & $4(2.1)$ & $7(0.9)$ & $3(0.6)$ & $4(1.4)$ \\
\hline Cytokines & $310(26.7)$ & $72(18.7)$ & 70 (36.6) & $2(1.0)$ & $238(30.7)$ & $228(45.9)$ & $10(3.6)$ \\
\hline - Interferon $\alpha-2 \beta$ & 309 (26.6) & $72(18.7)$ & 70 (36.6) & $2(1.0)$ & $237(30.6)$ & $227(45.7)$ & $10(3.6)$ \\
\hline - Peginterferon $\alpha-2 \beta$ & $89(7.7)$ & $19(4.9)$ & $18(9.4)$ & $1(0.5)$ & $70(9.0)$ & $68(13.7)$ & $2(0.7)$ \\
\hline - Aldesleukin & $4(0.3)$ & $2(0.5)$ & $2(1.0)$ & - & $2(0.3)$ & $1(0.2)$ & $1(0.4)$ \\
\hline Chemotherapy & $111(9.6)$ & $21(5.4)$ & $10(5.2)$ & $11(5.6)$ & $90(11.6)$ & $39(7.8)$ & $51(18.3)$ \\
\hline - Temozolomide & $56(4.8)$ & $8(2.1)$ & $5(2.6)$ & $3(1.5)$ & $48(6.2)$ & $19(3.8)$ & $29(10.4)$ \\
\hline - Fluorouracil & $6(0.5)$ & $1(0.3)$ & - & $1(0.5)$ & $5(0.6)$ & $5(1.0)$ & - \\
\hline - Carboplatin & $44(3.8)$ & $8(2.1)$ & $5(2.6)$ & $3(1.5)$ & $36(4.6)$ & $18(3.6)$ & $18(6.5)$ \\
\hline - PaclitaxelI & $46(4)$ & $9(2.3)$ & $3(1.6)$ & $6(3.1)$ & $37(4.8)$ & $15(3.0)$ & $22(7.9)$ \\
\hline - Dacarbazine & $13(1.1)$ & $2(0.5)$ & - & $2(1.0)$ & $11(1.4)$ & $2(0.4)$ & $9(3.2)$ \\
\hline - Cisplatin & $7(0.6)$ & - & - & - & $7(0.9)$ & $5(1.0)$ & $2(0.7)$ \\
\hline - Cyclophosphamide & - & - & - & - & - & - & - \\
\hline - Other chemotherapy & $4(0.3)$ & $1(0.2)$ & - & $1(0.5)$ & $3(0.4)$ & $3(0.6)$ & - \\
\hline \multicolumn{8}{|c|}{$\begin{array}{l}\text { Data are presented as } n(\%) \text {. } \\
\text { †FDA approval dates of checkpoint inhibitors for melanoma treatment: ipilimumab, March 2011; pembrolizumab, September 2014; nivolumab, December } 2014 . \\
\text { †FDA approval dates of BRAF/MEK inhibitors for melanoma treatment: dabrafenib, May 2013; trametinib, May 2013; vemurafenib, August } 2011 \text {; cobimetinib, November } 2015 . \\
\text { \$Other targeted therapies included bevacizumab, bortezomib, rituximab, and ruxolitinib phosphate. } \\
\mathbb{I}_{\text {And paclitaxel protein-bound. }} \\
\text { FDA: US Food and Drug Administration. }\end{array}$} \\
\hline
\end{tabular}

BRAF/MEK inhibitors may be used for patients with $B R A F^{M u t}$ tumors who have a higher comorbidity burden, including brain metastases [19], recent analyses suggest that checkpoint inhibitors may be the preferred first-line option for patients with brain metastases, given that they appear to result in comparable overall response rates but higher rates of durable intracranial responses [26]. In stage III melanoma, checkpoint inhibitors were used more commonly in $B R A F^{M u t}$ compared with $B R A F^{W t}$ melanomas, although the reasons for this could not be determined by this analysis. By contrast, in stage IV melanomas, the overall use of checkpoint inhibitors in any line of therapy was less in $B R A F^{M u t}$ compared with $B R A F^{W t}$ melanomas. Chemotherapy was most widely used in patients with $B R A F^{W t}$ stage IV melanoma compared with $B R A F^{W t}$ stage III or $B R A F^{M u t}$ melanoma, presumably because of a lack of other viable options for such late-stage melanoma.

Results from this study indicate that treatment patterns for advanced melanoma changed from 2011 to 2017. Since their approval, checkpoint inhibitors and BRAF/MEK inhibitors are continuing to become more widely prescribed, whereas cytokines and chemotherapy are being used less. It is unsurprising that the use of interferon $\alpha-2 \beta$ declined after 2014, given the approval of ipilimumab in 2015 as an adjuvant therapy for melanoma. Additionally, the increased uses of ipilimumab + nivolumab and nivolumab alone after 2015 were also foreseeable, given that the escalation corresponded with the publication of the initial results from the CheckMate 067 trial that demonstrated improved outcomes with nivolumab + ipilimumab or nivolumab alone versus ipilimumab alone in 
patients with metastatic melanoma [27]. These practices align with the current National Comprehensive Cancer Network [28] and European Society for Medical Oncology [29] clinical practice guidelines, which recommend the use of anti-PD-1 inhibitors (i.e., nivolumab and pembrolizumab) or nivolumab + ipilimumab as first-line therapy for all advanced melanoma patients, or combination therapy with BRAF/MEK inhibitors for patients with BRAF Mut melanoma [28,29]. Recommended second-line options include the use of checkpoint inhibitors and BRAF/MEKtargeted therapies for those with $B R A F^{M u t}$ disease, in addition to the use of systemic cytotoxic agents and high-dose IL-2 [28,29]. Of note, despite the recommendations to use BRAF/MEK inhibitors in combination as first-line treatment options (i.e., dabrafenib + trametinib, vemurafenib + cobimetinib, or encorafenib + binimetinib) [28,29], some agents continue to be used as monotherapies. Additionally, although BRAF inhibitors are only recommended for patients with $B R A F^{M u t}$ melanoma [28,29], their use as treatment for $B R A F^{W t}$ disease was observed in a small percentage of patients. Additionally, the combination of dabrafenib + trametinib was prescribed more frequently than cobimetinib + vemurafenib. Interestingly, in the adjuvant setting, in addition to the preapproval use of nivolumab, pembrolizumab use among these patients was observed.

New agents continue to be approved for melanoma, and, as such, ongoing surveillance of treatment patterns will be necessary to capture the continually changing landscape of treatment options and practices. For example, the approved use of the intralesional oncolytic virus talimogene laherparepvec (IMLYGIC@; Amgen Inc., CA, USA) as monotherapy for previously resected melanoma no longer amenable to surgical therapy is listed in the National Comprehensive Cancer Network guidelines, and these same guidelines also recommend the use of talimogene laherparepvec in combination with ipilimumab for patients with metastatic or unresectable disease. Talimogene laherparepvec and other local therapies are not reported here, although they may be used in combination with the systemic therapies reported in this study.

A notable strength of the current study was the large, heterogeneous population of patients with melanoma identified through the OSCER database. This population included oncology practices across the USA, thus representing geographic diversity as well as multiple EMR systems, payer types and clinical pathways. Patient-level data were captured systematically and electronically from the same EMR used by practitioners to track patient care, resulting in improved consistency. Nevertheless, data limitations prevent complete ascertainment of treatment that occurs outside of the outpatient clinic setting, and analyses were limited by unknown or missing information in some EMR data fields captured by the OSCER database. For example, we lacked melanoma substage biopsy data, which could have otherwise provided greater insight into the reasons behind the choices of adjuvant therapies. Additionally, each EMR only captured patients' treatment at one clinic; it is possible that the patients could have received further treatment at another location that would not be captured by this study. Furthermore, the OSCER database does not include patients from every region of the USA. Therefore, analyses may be limited by regional differences in treatment, and results from this study may not be generalizable to a broader population of patients with melanoma in the USA.

A similar analysis of patient EMRs was recently published that evaluated treatment patterns identified from the Flatiron Health database in adult patients with advanced melanoma who initiated first-line therapy between 1 January 2014 and 30 June 2016; however, that analysis included fewer patients for a smaller follow-up window than was reported in this study and focused primarily on the first-line setting [17]. Results from that analysis were similar to those observed here; overall, most patients $(>50 \%)$ received a checkpoint inhibitor as either monotherapy or as combination therapy for first- or second-line therapy [17]. For patients with $B R A F^{M u t}$ disease, BRAF inhibitors were the most commonly prescribed treatment [17]. Fewer patients with $B R A F^{W t}$ melanoma were noted to have received BRAF inhibitors in that study compared with this analysis [17].

The Flatiron Health database is a cloud-based database containing EMR data from $>265$ cancer clinics in the USA. The prescribing patterns captured by EMR databases, such as the Flatiron Health database, may be geographically limited and not representative of those at different types of clinical practice, including at academic medical centers.

Similar treatment patterns were also observed in a recent analysis of two US claims databases that evaluated patients with BRAF $F^{M u t}$ tumors with claims filed between 1 January 2014 and 30 June 2016 (Truven Health Analytics MarketScan, part of the IBM Watson Health ${ }^{T M}$ business, MI, USA), or 30 September 2016 (IQVIA, NC, USA) [19].

Truven Health Analytics MarketScan and the IQVIA health services databases contain claims data from approximately 100 employers and $>100$ US health plans, respectively. Similar to the geographic limitations noted for EMR databases, results from these two US claims databases are also limited in their generalizability to a broader population of patients with melanoma in the USA, given their regional focus. 
Overall, the results from this analysis show that checkpoint inhibitors have largely replaced other therapies in the first-line setting for advanced melanoma, including for patients with $B R A F^{M u t}$ melanoma. Additionally, the uses of BRAF inhibitor monotherapies are being replaced by BRAF/MEK combination inhibitors. However, although this comprehensive study provides a more thorough understanding of the treatment choices for patients with advanced melanoma and the changes in the therapeutic landscape over the years, there are many questions that remain unanswered that could further inform clinical practice. For example, treatment patterns by disease substage and patient age are still unknown, as are the time intervals between various lines of treatment.

Data from these analyses describe the changing treatment patterns in the real world and, when considered alongside efficacy and safety data, provide useful information to help guide treatment selections with a goal to seeking optimal patient outcomes. Further analyses should enable clinicians to maintain a current understanding of the evolving treatment landscape in melanoma.

\section{Conclusion}

In accordance with recent developments in the therapeutic landscape and with new treatment options available, checkpoint inhibitors have largely replaced other therapies in the first-line setting for treatment of advanced melanoma. For patients with $B R A F^{M u t}$ melanoma, major shifts also include BRAF inhibitors being replaced by $\mathrm{BRAF} / \mathrm{MEK}$ combination inhibitors. Overall, advancements in the field since 2011 have provided more treatment options for advanced melanoma, regardless of $B R A F$ mutation and stage.

\section{Supplementary data}

To view the supplementary data that accompany this paper please visit the journal website at: www.futuremedicine.com/doi/sup $\mathrm{pl} / 10.2217 / \mathrm{mmt}-2019-0013$

\section{Author contributions}

All authors contributed to the conception/design of the work, the analysis and interpretation of the work and drafting of the manuscript; provided final approval to be published; and agreed to be accountable for all aspects of the work to ensure accuracy and integrity.

\section{Financial \& competing interests disclosure}

S Shah, L Raskin and D Cohan are employees of and stockholders in Amgen Inc. O Hamid has served as a consultant for Amgen Inc., Novartis, Roche, Bristol-Meyers Squibb, Merck; has served on speakers bureaus for Amgen Inc., Bristol-Meyers Squibb, Genentech, Novartis, Array, and Sanofi-Regeneron; and has received institutional research funding from AstraZeneca, Bristol-Meyers Squibb, Celldex, Genentech Roche, Immunocore, Incyte, Merck, Merck Serono, Medlmmune, Novartis, Pfizer, Amgen Inc., Cytomx, lovance, Regeneron, and Polynoma. M Freeman has served as an advisory board consultant for Bristol-Meyers Squibb, Merck, Novartis, and Sanofi-Regeneron. The study was funded by Amgen Inc. The authors have no other relevant affiliations or financial involvement with any organization or entity with a financial interest in or financial conflict with the subject matter or materials discussed in the manuscript apart from those disclosed.

Medical writing support was provided by M Johnson (Complete Healthcare Communications, LLC, PA, USA) and was funded by Amgen Inc.

\section{Open access}

This work is licensed under the Attribution-NonCommercial-NoDerivatives 4.0 Unported License. To view a copy of this license, visit http://creativecommons.org/licenses/by-nc-nd/4.0/

\section{Data sharing statement}

Qualified researchers may request data from Amgen clinical studies. Complete details are available at the following: https://www. amgen.com/science/clinical-trials/clinical-data-transparency-practices/

\section{References}

Papers of special note have been highlighted as: $\bullet$ of interest; $\bullet \bullet$ of considerable interest

1. National Institutes of Health, National Cancer Institute. SEER cancer stat facts: melanoma of the skin 2018 (2019). https://seer.cancer.gov/statfacts/html/melan.html.

2. American Cancer Society. Cancer facts \& figures 2016 (2018). https://www.cancer.org/content/dam/cancer-org/research/cancer-factsand-statistics/annual-cancer-facts-and-figures/2016/cancer-facts-and-figures-2016.pdf.

3. Domingues B, Lopes JM, Soares P, Populo H. Melanoma treatment in review. Immunotargets Ther. 7, 35-49 (2018). 
4. Thomas NE, Edmiston SN, Alexander A et al. Association between NRAS and BRAF mutational status and melanoma-specific survival among patients with higher-risk primary melanoma. JAMA Oncol. 1(3), 359-368 (2015).

5. Barbour AP, Tang $\mathrm{YH}$, Armour $\mathrm{N}$ et al. BRAF mutation status is an independent prognostic factor for resected stage IIIB and IIIC melanoma: implications for melanoma staging and adjuvant therapy. Eur. J. Cancer 50(15), 2668-2676 (2014).

6. Yervoy ${ }^{\circledR}$ (ipilimumab). Full prescribing information. Bristol-Myers Squibb, Princeton, NJ, USA (2019).

7. KEYTRUDA $^{\circledR}$ (pembrolizumab). Full prescribing information. Merck \& Co., Inc., Whitehouse Station, NJ, USA (2019).

8. Opdivo $^{\circledR}$ (nivolumab). Full prescribing information. Bristol-Myers Squibb, Princeton, NJ, USA (2019).

9. Saraceni MM, Khushalani NI, Jarkowski A 3rd. Immunotherapy in melanoma: recent advances and promising new therapies. J. Pharm. Pract. 28(2), 193-203 (2015).

10. IMLYGIC $^{\circledR}$ (talimogene laherparepvec). Full prescribing information. Amgen Inc., Thousand Oaks, CA, USA (2019).

11. ZELBORAF ${ }^{\circledR}$ (vemurafenib). Full prescribing information. Genentech USA, Inc., San Francisco, CA, USA (2017).

12. TAFINLAR ${ }^{\circledR}$ (dabrafenib). Full prescribing information. Novartis Pharmaceuticals Corporation, East Hanover, NJ, USA (2017).

13. BRAFTOVI $^{\text {TM }}$ (encorafenib). Full prescribing information. Array BioPharma, Inc., Boulder, CO, USA (2018).

14. MEKINIST ${ }^{\circledR}$ (trametinib). Full prescribing information. GlaxoSmithKline, Research Triangle Park, NC, USA (2018).

15. COTELLIC ${ }^{\circledR}$ (cobimetinib). Full prescribing information. Genentech, Inc., South San Francisco, CA, USA (2018).

16. MEKTOVI $^{\text {TM }}$ (binimetinib). Full prescribing information. Array Biopharma, Inc., Boulder, CO, USA (2018).

17. Whitman ED, Liu FX, Cao X, Diede SJ, Haiderali A, Abernethy AP. Treatment patterns and outcomes for patients with advanced melanoma in US oncology clinical practices. Future Oncol. 15(5), 459-471 (2019).

-• Retrospective electronic medical record (EMR) analysis of first-line treatment patterns and outcomes for patients with advanced melanoma by $B R A F$ mutation status from the Flatiron Health database from 2014 to 2017 in the USA.

18. Hassel JC, Buder-Bakhaya K, Bender $\mathrm{C}$ et al. Progression patterns under BRAF inhibitor treatment and treatment beyond progression in patients with metastatic melanoma. Cancer Med. 7(1), 95-104 (2018).

-. Retrospective analysis of treatment patterns and disease progression in patients with $B R A F$-mutant metastatic melanoma who progressed after BRAF inhibitor monotherapy between 2010 and 2015. Treatment after progression did not affect overall survival.

19. Ghate $\mathrm{S}$, Ionescu-Ittu R, Burne $\mathrm{R}$ et al. Patterns of treatment and $B R A F$ testing with immune checkpoint inhibitors and targeted therapy in patients with metastatic melanoma presumed to be BRAF positive. Melanoma Res. 29(3), 301-310 (2019).

-. Treatment patterns based on BRAF mutation status testing from 2014 to 2016 using patient claims data in the USA. BRAF/MEK inhibitors may be used for patients with $B R A F$ mutations who have a higher comorbidity burden.

20. Mohr P, Ascierto P, Arance A et al. Real-world treatment patterns and outcomes among metastatic cutaneous melanoma patients treated with ipilimumab. J. Eur. Acad. Dermatol. Venereol. 32(6), 962-971 (2018).

- Uses EMRs to assess treatment patterns in melanoma outside of the USA.

21. Sassolas B, Leccia MT, Godard C et al. Real-world treatment patterns and clinical outcomes in advanced cutaneous melanoma patients in France. J. Eur. Acad. Dermatol. Venereol. 32(4), 587-594 (2018).

- Uses EMRs to assess treatment patterns in melanoma outside of the USA.

22. Hecht JR, Pless M, Cubillo A et al. Early safety from a Phase I, multicenter, open-label clinical trial of talimogene laherparepvec (T-VEC) injected into liver tumors. J. Clin. Oncol. 36(Suppl. 4S), Abstract 438 (2018).

23. Jones C, Zhao Z, Barber B, Bagijn M, Corrie P, Saltman D. Treatment patterns in advanced melanoma: findings from a survey of European oncologists. Eur. J. Cancer Care (Engl.) 24(6), 862-866 (2015).

24. Khozin S, Carson KR, Zhi J et al. Real-world outcomes of patients with metastatic non-small cell lung cancer treated with programmed cell death protein 1 inhibitors in the year following U.S. regulatory approval. Oncologist doi:10.1634/theoncologist.2018-0307 (2018) (Epub ahead of print).

25. Khozin S, Abernethy AP, Nussbaum NC et al. Characteristics of real-world metastatic non-small cell lung cancer patients treated with nivolumab and pembrolizumab during the year following approval. Oncologist 23(3), 328-336 (2018).

26. Tawbi HA, .Boutros C, Kok D, Robert C, Mcarthur G. New era in the management of melanoma brain metastases. Am. Soc. Clin. Oncol. Educ. Book 38, 741-750 (2018).

27. Larkin J, Chiarion-Sileni V, Gonzalez R et al. Combined nivolumab and ipilimumab or monotherapy in untreated melanoma. $N$. Engl. J. Med. 373(1), 23-34 (2015).

28. National Comprehensive Cancer Network. NCCN clinical practice guidelines in oncology (NCCN guidelines ${ }^{\circledR}$ ): cutaneous melanoma version 1.2019 (2019). https://www.nccn.org/professionals/physician_gls/pdf/cutaneous_melanoma.pdf

- Comprehensive treatment guidelines describing sequential treatment decisions for cutaneous melanoma in the USA.

29. Dummer R, Hauschild A, Lindenblatt N, Pentheroudakis G, Keilholz U, ESMO Guidelines Committee. Cutaneous melanoma: ESMO clinical practice guidelines for diagnosis, treatment and follow-up. Ann. Oncol. 26(Suppl. 5), v126-v132 (2015).

- Treatment guidelines for the diagnosis and treatment of cutaneous melanoma as well as follow-up recommendations and monitoring. 